\title{
A Case Report of Widespread Melanoma with a Complete Response to Pembrolizumab
}

\author{
${ }^{1}$ Department of Oncology, Vaasa Central Hospital, Finland \\ ${ }^{2}$ Turku University, Finland \\ ${ }^{3}$ Tema Cancer, Karolinska University Hospital, Sweden \\ ${ }^{4}$ Tampere University, Finland \\ ${ }^{5}$ Department of Radiology, Vaasa Central Hospital, Finland
}

Natalja Eigeliene $^{1,2 *}$, Heidi Andersèn ${ }^{1,3,4}$, Ravichandra Ravi ${ }^{1}$, Anna Laaksonen ${ }^{2,5}$ and Antti Jekunen ${ }^{1,2}$

Submission: April 14, 2021; Published: May 10, 2021

*Corresponding author: Natalja Eigeliene, University of Turku, Vaasa Central Hospital, Oncology, Vaasa, Hietalahdenkatu 2-4, 65130 Vaasa, Finland

\begin{abstract}
Melanoma is an aggressive disease that accounts for approximately $2 / 3$ of skin cancer-related deaths. In the past, treatment options for patients with advanced melanoma have been limited, with a poor response rate and failure to improve overall survival (OS). Immunotherapy is a promising treatment that has been shown to improve OS and, in many cases, produce durable remission in patients with advanced melanoma. Here, we report a case of a patient with widespread malignant melanoma at progression after biochemotherapy who achieved a durable complete response to pembrolizumab immunotherapy without major side effects. The patient has been progression-free and treatment-free for four years. This case adds to the evidence in support of early referral and initiation of pembrolizumab for patients who can tolerate it, with the intent to produce a sustained treatment-free response and preserved quality of life.
\end{abstract}

Keywords: Melanoma; Pembrolizumab; Immunotherapy; DOBS; Complete response

Abbreviation: DOBS: Lomustine, Bleomycin, Vincristine, Dacarbazine; I: Interferon; CT: Computer Tomography; MRI: Magnetic Resonance Imaging; FDG: Fluorodeoxyglucose; PET: Positron Emission Tomography; iv: Intravenous; sc: Subcutaneous

\section{Introduction}

Cutaneous melanoma, a type of skin cancer, has risen in incidence in recent years, according to the NCCN guidelines for cutaneous melanoma [1]. Unresectable or metastatic malignant melanoma has until recently been very difficult to treat. New therapies, including targeted therapies and immunotherapy, have been developed and implemented according to clinical guidelines [1]. Immunotherapy is an essential part of the treatment of metastatic and unresectable malignant melanoma [1]. Nevertheless, more research is needed to establish the optimal administration, treatment combination, and duration of therapy for different immunotherapeutic agents.

\section{Case Report}

We present the case of a 70-year-old Caucasian man who reported with worsening pain in the lower back and left hip in 2016. He was an ex-smoker with no history of trauma to the back. The patient had psoriasis that had been in remission for several years. First, the patient sought treatment from a private doctor. Spine and SI-joint magnetic resonance imaging (MRI) was performed and revealed bone metastases. Thus, the patient underwent a body computer tomography (CT) scan, which showed multiple metastases in the thyroid gland, lungs, mediastinum, liver, and vertebrae Th4 and Th7 (Figure 1). A biopsy from the sacrum was obtained, and histology confirmed melanoma without BRAF mutation. The primary origin of the melanoma was not found.

Radiotherapy was given due to pain in the left hip (10 x 3Gy). Chemotherapy was then initiated with DOBS (lomustine, bleomycin, vincristine, dacarbazine)-interferon (I), which caused substantial side effects, such as weight loss, fatigue, nausea, and vomiting. Additionally, due to bone metastases, denosumab $120 \mathrm{mg}$ 
was given subcutaneously (sc). Three cycles of DOBS-I were given before treatment response was evaluated with a whole-body CT scan. Unfortunately, in the initial scan, the tumor was found to have progressed in the liver, mediastinum, and lungs. Thus, a decision to start second-line treatment with pembrolizumab was made.

Two hundred milligrams of pembrolizumab were given intravenously (iv) every third week. The patient had mild treatment-related side effects. After the second treatment with pembrolizumab, an itchy rash appeared on both lower extremities; a dermatologist diagnosed the rash as psoriasis. Local application of an anti-psoriatic cream comprised of calcipotriol and betamethasone was recommended and alleviated the rash. After the third pembrolizumab treatment in 2017, a whole-body CT scan revealed an excellent response to the treatment. Together, ten treatments were given triweekly, and FDG-PET-CT was performed (Figure 1). The patient achieved a complete response. Thus, pembrolizumab and denosumab were discontinued, and follow-up was initiated, including clinical examination, blood analyses, and regular CT scans. In spring 2020, the left hip joint was replaced by a prosthetic implant without any complications. The patient has attended regular follow-up visits at the dermatology and oncology clinics. To date, the patient has remained stable, with no signs of progression on the last CT scan on 2/2021 (Figure 1).

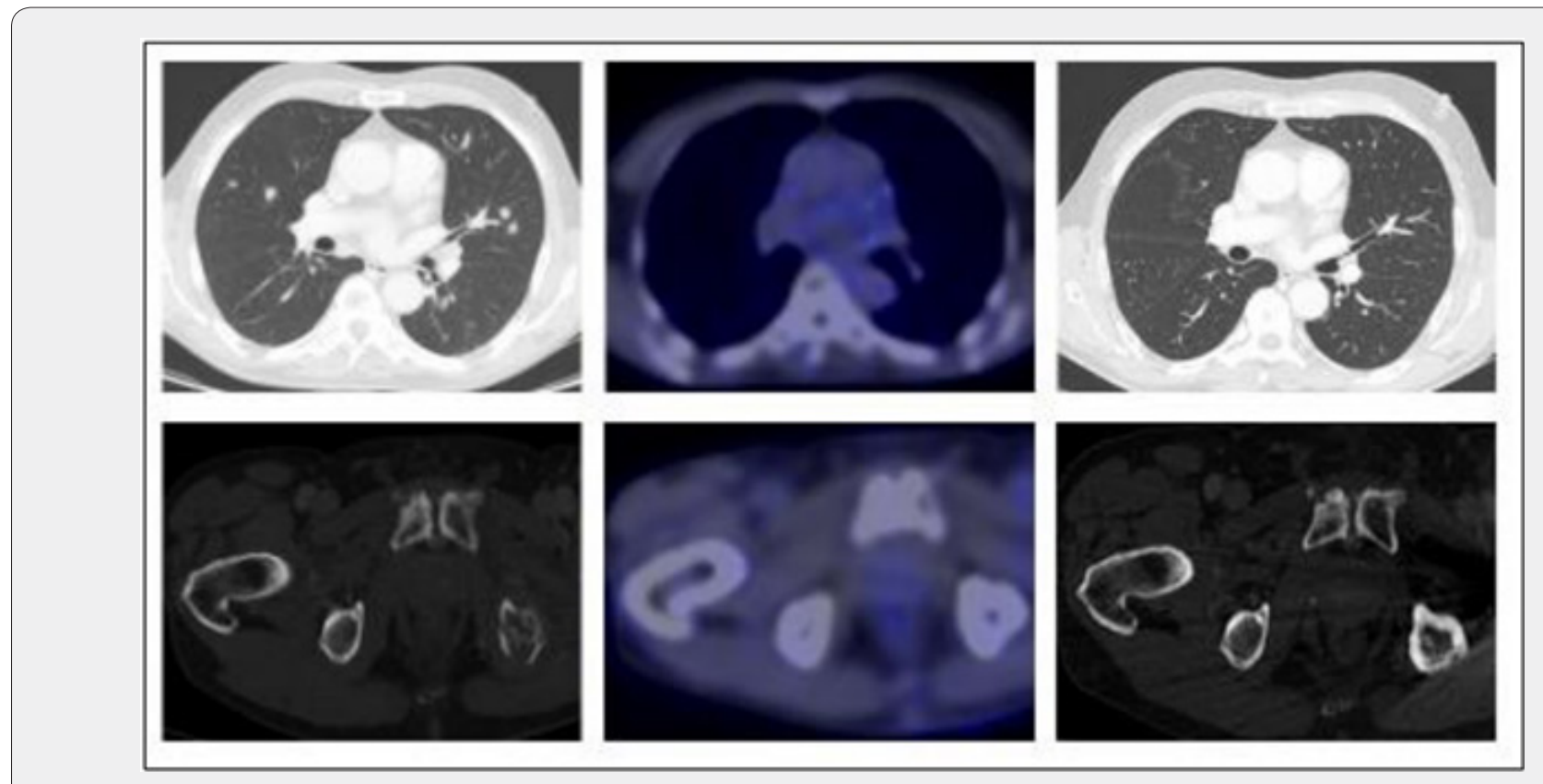

Figure 1: On the left side CT scan from 2016, in the middle negative FDG-PET scan from 2017 and on the right-side CT scan from 2021 that shows no progress in pulmonary or skeletal metastasis compared to 2017.

\section{Discussion}

Despite many years of research and some advances, metastatic malignant melanoma remains a significant challenge for modern medicine and is still regarded as incurable [2,3]. In our patient with widespread malignant melanoma, a durable (4 years) complete response to pembrolizumab immunotherapy as a second-line therapy for progression after radiotherapy and biochemotherapy was achieved. Follow-up is ongoing, without treatment, and no signs of cancer have been detected since 2017. There is a chance that this patient's metastatic melanoma has been cured; however, it is too early to make that conclusion. These findings highlight the changes in active cancer treatments for solid tumors from life-extending treatments to curative therapies that can induce remission and potentially lead to cure, which requires life-long sustained remission [4].

A recent Cochrane analysis [5] demonstrated that immune checkpoint inhibitors improved progression-free survival rates compared to chemotherapy (HR $0.30,95 \%$ CI 0.17 to 0.51 ) and that patients who received chemotherapy had worse PFS rates than those who received a combination of immune checkpoint inhibitors (HR 2.81, 95\% CI 1.54 to 5.11) for melanoma.

The CR rate for chemotherapy is typically low, as shown in a meta-analysis including 68 chemotherapy trials for cancer in which the average CR rate was found to be $7.41 \%$ (5-10\%) in solid tumors, e.g., colon, breast prostate, and melanoma (excluding testis cancer and pediatric leukemia) [6]. The CR rate has also been found to be low in patients with melanoma treated with single immunotherapeutics with a CR rate of $7.6 \%$ achieved with nivolumab [7] 1.7\% with ipilimumab and 5-6.1\% with pembrolizumab [7]. However, the CR rate improved up to $20 \%$ with pembrolizumab in a prolonged study in which five-year responses [8]. Currently, it is acceptable to discontinue checkpoint inhibitor treatment for patients who have achieved CR after six months of therapy, since the five-year risk of relapse is estimated to be less than $10 \%$ [8]. 
A durable complete response can be maintained after discontinuation of pembrolizumab in patients with metastatic melanoma. FDG PET/CT imaging can be used to assess tumor response to immunotherapy $[9,10]$. Our case report describes a clinical example of the successful use of PET CT imaging, as after the initial negative examination, the patient's melanoma has not relapsed; however, the optimal timing and utilization of FDG PET/ CT imaging for decision making needs to be further investigated in prospective studies.

\section{Informed Consent}

The patient provided oral and written consent to be included in the case study.

\section{References}

1. NCCN Clinical Practice Guidelines in Oncology Cutaneous melanoma (2020).

2. (2020) Key Statistics for Melanoma Skin Cancer. In: The American Cancer Society Medical and Editorial Content Team

3. Cherny NI, Dafni U, Bogaerts J, Latino NJ, Pentheroudakis G, et al. (2017) ESMO-Magnitude of Clinical Benefit Scale version 1.1. Ann Oncol 28(10): 2340-2366.
4. Neugut AI, Prigerson HG (2017) Curative, Life-Extending, and Palliative Chemotherapy: New Outcomes Need New Names. Oncologist 22(8): 883-885.

5. Pasquali S, Hadjinicolaou AV, Sileni VC, Rossi CR, Mocellin S (2018) Systemic treatments for metastatic cutaneous melanoma. Cochrane Database Syst Rev 2(2): CD011123.

6. Ashdown ML, Robinson AP, Yatomi Clarke SL, Ashdown ML, Allison A, et al. (2015) Chemotherapy for Late-Stage Cancer Patients: MetaAnalysis of Complete Response Rates. F1000Res 4: 232.

7. Robert C, Long GV, Brady B, Dutriaux C, Maio M, Mortier L, et al. (2015) Nivolumab in previously untreated melanoma without BRAF mutation. N Engl J Med 372(4): 320-330.

8. Robert C, Ribas A, Hamid O, Daud A, Wolchok JD, et al. (2018) Durable Complete Response After Discontinuation of Pembrolizumab in Patients with Metastatic Melanoma. J Clin Oncol 36(17): 1668-1674.

9. Aide N, Hicks RJ, Le Tourneau C, Lheureux S, Fanti S, et al. (2019) FDG $\mathrm{PET} / \mathrm{CT}$ for assessing tumour response to immunotherapy : Report on the EANM symposium on immune modulation and recent review of the literature. Eur J Nucl Med Mol Imaging 46(1): 238-250.

10. Lang D, Wahl G, Poier N, Graf S, Kiesl D, et al. (2020) Impact of PET/CT for Assessing Response to Immunotherapy-A Clinical Perspective. J Clin Med 9(11): 3483.

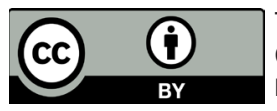

This work is licensed under Creative Commons Attribution 4.0 License DOI: 10.19080/JOJCS.2021.12.555837 